FORMALIZED MATHEMATICS

Vol. 21, No. 2, Pages 103-113, 2013

DOI: $10.2478 /$ forma-2013-0012
VERSIT $\Lambda$

degruyter.com/view/j/forma

\title{
Polygonal Numbers
}

\author{
Adam Grabowski \\ Institute of Informatics \\ University of Białystok \\ Akademicka 2, 15-267 Białystok \\ Poland
}

\begin{abstract}
Summary. In the article the formal characterization of triangular numbers (famous from [15] and words "EYPHKA! num $=\Delta+\Delta+\Delta$ ") [17] is given. Our primary aim was to formalize one of the items (\#42) from Wiedijk's Top 100 Mathematical Theorems list 33], namely that the sequence of sums of reciprocals of triangular numbers converges to 2. This Mizar representation was written in 2007. As the Mizar language evolved and attributes with arguments were implemented, we decided to extend these lines and we characterized polygonal numbers.

We formalized centered polygonal numbers, the connection between triangular and square numbers, and also some equalities involving Mersenne primes and perfect numbers. We gave also explicit formula to obtain from the polygonal number its ordinal index. Also selected congruences modulo 10 were enumerated. Our work basically covers the Wikipedia item for triangular numbers and the Online Encyclopedia of Integer Sequences (http://oeis.org/A000217).

An interesting related result [16] could be the proof of Lagrange's four-square theorem or Fermat's polygonal number theorem [32.
\end{abstract}

MSC: 11E25 03B35

Keywords: triangular number; polygonal number; reciprocals of triangular numbers

MML identifier: NUMPOLY1, version: 8.1.02 5.17.1179

The notation and terminology used in this paper have been introduced in the

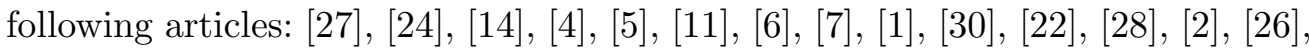
[21], [3], 8], 13], 34], 18], 35], [9], [19], 20], 25], 29], 31], and [10].

\section{Preliminaries}

The scheme LNatRealSeq deals with a unary functor $\mathcal{F}$ yielding a real number and states that 
(Sch. 1) there exists a sequence $s_{3}$ of real numbers such that for every natural number $n, s_{3}(n)=\mathcal{F}(n)$ and for every sequences $s_{1}, s_{2}$ of real numbers such that for every natural number $n, s_{1}(n)=\mathcal{F}(n)$ and for every natural number $n, s_{2}(n)=\mathcal{F}(n)$ holds $s_{1}=s_{2}$.

Now we state the proposition:

(1) Let us consider non zero natural numbers $n$, $a$. Then $1 \leqslant a \cdot n$.

Let $n$ be an integer. One can verify that $n \cdot(n-1)$ is even and $n \cdot(n+1)$ is even.

Now we state the proposition:

(2) Let us consider an even integer $n$. Then $\frac{n}{2}$ is an integer.

Let $n$ be an even natural number. One can verify that $\frac{n}{2}$ is natural.

Let $n$ be an odd natural number. One can verify that $n-1$ is natural.

Let us note that $n-1$ is even.

In this paper $n, s$ denote natural numbers.

Now we state the propositions:

(3) $n \bmod 5=0$ or $\ldots$ or $n \bmod 5=4$.

(4) Let us consider a natural number $k$. If $k \neq 0$, then $n \equiv n \bmod k(\bmod k)$.

(5) $n \equiv 0(\bmod 5)$ or $\ldots$ or $n \equiv 4(\bmod 5)$. The theorem is a consequence of (3) and (4).

Now we state the propositions:

(6) $n \cdot n+n \not \equiv 4(\bmod 5)$.

(7) $n \cdot n+n \not \equiv 3(\bmod 5)$.

Now we state the propositions:

(8) $n \bmod 10=0$ or $\ldots$ or $n \bmod 10=9$.

(9) $n \equiv 0(\bmod 10)$ or $\ldots$ or $n \equiv 9(\bmod 10)$. The theorem is a consequence of (8) and (4).

Note that every natural number which is non trivial is also 2 or greater and every natural number which is 2 or greater is also non trivial and every natural number which is 4 or greater is also 3 or greater and non zero and every natural number which is 4 or greater is also non trivial and there exists a natural number which is 4 or greater and there exists a natural number which is 3 or greater.

\section{TRiangular Numbers}

Let $n$ be a natural number. The functor Triangle $n$ yielding a real number is defined by the term

(Def. 1) $\sum \operatorname{idseq}(n)$.

Let $n$ be a number. We say that $n$ is triangular if and only if

(Def. 2) There exists a natural number $k$ such that $n=$ Triangle $k$. 
Let $n$ be a zero number. Let us observe that Triangle $n$ is zero.

Now we state the propositions:

(10) Triangle $(n+1)=$ Triangle $n+(n+1)$. Proof: Define $\mathcal{P}$ [natural number $] \equiv$ Triangle $\$_{1}+\left(\$_{1}+1\right)=$ Triangle $\left(\$_{1}+1\right)$. For every natural number $k$ such that $\mathcal{P}[k]$ holds $\mathcal{P}[k+1]$ by [5, (51)], [9, (74)]. For every natural number $n, \mathcal{P}[n]$ from [2, Sch. 2].

(11) Triangle $1=1$.

(12) Triangle $2=3$.

(13) Triangle $3=6$.

(14) Triangle $4=10$. The theorem is a consequence of (10) and (13).

(15) Triangle $5=15$. The theorem is a consequence of (10) and (14).

(16) Triangle $6=21$. The theorem is a consequence of (10) and (15).

(17) Triangle $7=28$. The theorem is a consequence of (10) and (16).

(18) Triangle $8=36$. The theorem is a consequence of (10) and (17).

(19) Triangle $n=\frac{n \cdot(n+1)}{2}$. The theorem is a consequence of (10). Proof: Define $\mathcal{P}$ [natural number] $\equiv$ Triangle $\$_{1}=\frac{\$_{1} \cdot\left(\$_{1}+1\right)}{2}$. For every natural number $k$ such that $\mathcal{P}[k]$ holds $\mathcal{P}[k+1]$. For every natural number $n, \mathcal{P}[n]$ from [2, Sch. 2].

(20) Triangle $n \geqslant 0$. The theorem is a consequence of (19).

Let $n$ be a natural number. Observe that Triangle $n$ is non negative.

Let $n$ be a non zero natural number. Let us note that Triangle $n$ is positive. Let $n$ be a natural number. Observe that Triangle $n$ is natural.

Now we state the proposition:

(21) Triangle $\left(n-^{\prime} 1\right)=\frac{n \cdot(n-1)}{2}$. The theorem is a consequence of (1) and (19).

One can check that every number which is triangular is also natural and there exists a number which is triangular and non zero.

Let us consider a triangular number $n$. Now we state the propositions:

(22) $n \not \equiv 7(\bmod 10)$.

(23) $n \not \equiv 9(\bmod 10)$.

(24) $n \not \equiv 2(\bmod 10)$.

(25) $n \not \equiv 4(\bmod 10)$.

Now we state the proposition:

(26) Let us consider a triangular number $n$. Then

(i) $n \equiv 0(\bmod 10)$, or

(ii) $n \equiv 1(\bmod 10)$, or

(iii) $n \equiv 3(\bmod 10)$, or

(iv) $n \equiv 5(\bmod 10)$, or 
(v) $n \equiv 6(\bmod 10)$, or

(vi) $n \equiv 8(\bmod 10)$.

The theorem is a consequence of (9), (24), (25), (22), and (23).

\section{Polygonal Numbers}

Let $s, n$ be natural numbers. The functor $\operatorname{Polygon}(s, n)$ yielding an integer is defined by the term

(Def. 3) $\frac{n^{2} \cdot(s-2)-n \cdot(s-4)}{2}$.

Now we state the propositions:

(27) If $s \geqslant 2$, then Polygon $(s, n)$ is natural.

(28) $\operatorname{Polygon}(s, n)=\frac{(n \cdot(s-2)) \cdot(n-1)}{2}+n$.

Let $s$ be a natural number and $x$ be an element. We say that $x$ is $s$-gonal if and only if

(Def. 4) There exists a natural number $n$ such that $x=\operatorname{Polygon}(s, n)$.

We say that $x$ is polygonal if and only if

(Def. 5) There exists a natural number $s$ such that $x$ is $s$-gonal.

Now we state the propositions:

(29) $\operatorname{Polygon}(s, 1)=1$.

(30) $\operatorname{Polygon}(s, 2)=s$.

Let $s$ be a natural number. Note that there exists a number which is $s$-gonal.

Let $s$ be a non zero natural number. One can verify that there exists a number which is non zero and $s$-gonal.

Let $s$ be a natural number. One can verify that every number which is $s$-gonal is also real.

Let $s$ be a non trivial natural number. Let us observe that every number which is $s$-gonal is also natural.

Now we state the proposition:

(31) $\operatorname{Polygon}(s, n+1)-\operatorname{Polygon}(s, n)=(s-2) \cdot n+1$.

Let $s$ be a natural number and $x$ be an $s$-gonal number.

The functor IndexPoly $(s, x)$ yielding a real number is defined by the term

(Def. 6) $\frac{\left(\sqrt{(8 \cdot s-16) \cdot x+(s-4)^{2}}+s\right)-4}{2 \cdot s-4}$.

Let us consider a non zero natural number $s$ and a non zero $s$-gonal number $x$. Now we state the propositions:

(32) If $x=\operatorname{Polygon}(s, n)$, then $(8 \cdot s-16) \cdot x+(s-4)^{2}=((2 \cdot n) \cdot(s-2)-(s-4))^{2}$.

(33) If $s \geqslant 4$, then $(8 \cdot s-16) \cdot x+(s-4)^{2}$ is square.

(34) If $s \geqslant 4$, then $\operatorname{IndexPoly}(s, x) \in \mathbb{N}$. 
Now we state the propositions:

(35) Let us consider a non trivial natural number $s$ and an $s$-gonal number $x$. Then $0 \leqslant(8 \cdot s-16) \cdot x+(s-4)^{2}$.

(36) Let us consider an odd natural number $n$. If $s \geqslant 2$, then $n \mid \operatorname{Polygon}(s, n)$.

\section{Centered Polygonal Numbers}

Let $s, n$ be natural numbers. The functor $\operatorname{CentPoly}(s, n)$ yielding an integer is defined by the term

(Def. 7$) \frac{s \cdot n}{2} \cdot(n-1)+1$.

Let $s$ be a natural number and $n$ be a non zero natural number. One can verify that CentPoly $(s, n)$ is natural.

Now we state the propositions:

(37) $\operatorname{CentPoly}(0, n)=1$.

(38) $\operatorname{CentPoly}(s, 0)=1$.

(39) $\operatorname{CentPoly}(s, n)=s \cdot \operatorname{Triangle}\left(n-^{\prime} 1\right)+1$. The theorem is a consequence of $(21)$.

\section{On the Connection between Triangular and Other Polygonal NumBERS}

Now we state the propositions:

(40) Triangle $n=\operatorname{Polygon}(3, n)$. The theorem is a consequence of (19).

(41) Let us consider an odd natural number $n$. Then $n \mid$ Triangle $n$. The theorem is a consequence of (36) and (40).

(42) Triangle $n \leqslant$ Triangle $(n+1)$. The theorem is a consequence of $(10)$.

(43) Let us consider a natural number $k$. If $k \leqslant n$, then Triangle $k \leqslant$ Triangle $n$. The theorem is a consequence of (42). Proof: Consider $i$ being a natural number such that $n=k+i$. Define $\mathcal{P}$ [natural number] $\equiv$ for every natural number $n$, Triangle $n \leqslant$ Triangle $\left(n+\$_{1}\right)$. For every natural number $k$ such that $\mathcal{P}[k]$ holds $\mathcal{P}[k+1]$. For every natural number $n, \mathcal{P}[n]$ from [2, Sch. 2].

(44) $n \leqslant$ Triangle $n$. The theorem is a consequence of (10). Proof: Define $\mathcal{P}$ [natural number] $\equiv \$_{1} \leqslant$ Triangle $\$_{1}$. For every natural number $k$ such that $\mathcal{P}[k]$ holds $\mathcal{P}[k+1]$ by [2, (11)]. For every natural number $n, \mathcal{P}[n]$ from [2, Sch. 2].

(45) Let us consider a non trivial natural number $n$. Then $n<$ Triangle $n$. The theorem is a consequence of (12) and (10). Proof: Define $\mathcal{P}$ [natural number $] \equiv \$_{1}<$ Triangle $\$_{1}$. For every non trivial natural number $k$ such 
that $\mathcal{P}[k]$ holds $\mathcal{P}[k+1]$ by [2, (16)]. For every non trivial natural number $n, \mathcal{P}[n]$ from [23, Sch. 2].

(46) If $n \neq 2$, then Triangle $n$ is not prime. The theorem is a consequence of (11), (41), (45), and (19).

Let $n$ be a 3 or greater natural number. Observe that Triangle $n$ is non prime and every 4 or greater natural number which is triangular is also non prime.

Let $s$ be a 4 or greater non zero natural number and $x$ be a non zero $s$-gonal number. Note that IndexPoly $(s, x)$ is natural.

Now we state the propositions:

(47) Let us consider a 4 or greater natural number $s$ and a non zero $s$-gonal number $x$. If $s \neq 2$, then $\operatorname{Polygon}(s, \operatorname{IndexPoly}(s, x))=x$. The theorem is a consequence of (35).

(48) 36 is square and triangular. The theorem is a consequence of (19).

Let $n$ be a natural number. One can check that $\operatorname{Polygon}(3, n)$ is natural.

Observe that Polygon $(3, n)$ is triangular.

Now we state the propositions:

(49) Polygon $(s, n)=(s-2)$. Triangle $\left(n-^{\prime} 1\right)+n$. The theorem is a consequence of (21).

(50) Polygon $(s, n)=(s-3) \cdot$ Triangle $\left(n-^{\prime} 1\right)+$ Triangle $n$. The theorem is a consequence of $(21)$ and (19).

(51) $\operatorname{Polygon}(0, n)=n \cdot(2-n)$.

(52) $\operatorname{Polygon}(1, n)=\frac{n \cdot(3-n)}{2}$.

(53) $\operatorname{Polygon}(2, n)=n$.

Let $s$ be a non trivial natural number and $n$ be a natural number. Observe that Polygon $(s, n)$ is natural.

One can check that Polygon $(4, n)$ is square and every natural number which is 3-gonal is also triangular and every natural number which is triangular is also 3-gonal and every natural number which is 4-gonal is also square and every natural number which is square is also 4 -gonal.

Now we state the propositions:

(54) Triangle $\left(n-^{\prime} 1\right)+$ Triangle $n=n^{2}$. The theorem is a consequence of (19).

(55) Triangle $n+\operatorname{Triangle}(n+1)=(n+1)^{2}$. The theorem is a consequence of (19).

Let $n$ be a natural number. Observe that Triangle $n+\operatorname{Triangle}(n+1)$ is square.

Let us consider a non trivial natural number $n$. Now we state the propositions:

(56) $\frac{1}{3} \cdot$ Triangle $\left(3 \cdot n-^{\prime} 1\right)=\frac{n \cdot(3 \cdot n-1)}{2}$.

(57) Triangle $\left(2 \cdot n-^{\prime} 1\right)=\frac{n \cdot(4 \cdot n-2)}{2}$. 
Let $n, k$ be natural numbers. The functor $\operatorname{Power}_{\mathbb{N}}(n, k)$ yielding a finite sequence of elements of $\mathbb{R}$ is defined by

(Def. 8) (i) dom $i t=\operatorname{Seg} k$, and

(ii) for every natural number $i$ such that $i \in \operatorname{dom} i t$ holds $i t(i)=i^{n}$.

Now we state the proposition:

(58) Let us consider a natural number $k$. Then $\operatorname{Power}_{\mathbb{N}}(n, k+1)=\operatorname{Power}_{\mathbb{N}}(n, k)^{\frown}$ $\left\langle(k+1)^{n}\right\rangle$. Proof: $\operatorname{dom} \operatorname{Power}_{\mathbb{N}}(n, k+1)=\operatorname{dom}\left(\operatorname{Power}_{\mathbb{N}}(n, k) \bumpeq\left\langle(k+1)^{n}\right\rangle\right)$ by [4, (6), (40)]. For every natural number $l$ such that $l \in \operatorname{dom}_{\operatorname{Power}_{\mathbb{N}}}(n, k+$ 1) holds $\left(\operatorname{Power}_{\mathbb{N}}(n, k+1)\right)(l)=\left(\operatorname{Power}_{\mathbb{N}}(n, k)^{\frown}\left\langle(k+1)^{n}\right\rangle\right)(l)$ by [4, (1)], [2, (8)], [4, (6), (42)].

Let $n$ be a natural number. Let us observe that $\sum \operatorname{Power}_{\mathbb{N}}(n, 0)$ reduces to 0 . Now we state the propositions:

(59) (Triangle $n)^{2}=\sum$ Power $_{\mathbb{N}}(3, n)$. The theorem is a consequence of (19) and (58). Proof: Define $\mathcal{P}$ [natural number] $\equiv\left(\text { Triangle } \$_{1}\right)^{2}=\sum$ Power $_{\mathbb{N}}$ $\left(3, \$_{1}\right) . \mathcal{P}[0]$ by $[21$, (81)]. For every natural number $k$ such that $\mathcal{P}[k]$ holds $\mathcal{P}[k+1]$ by [21, (81), (7)], [12, (27)]. For every natural number $n, \mathcal{P}[n]$ from [2, Sch. 2].

(60) Let us consider a non trivial natural number $n$. Then Triangle $n+$ Triangle $\left(n-^{\prime} 1\right) \cdot$ Triangle $(n+1)=(\text { Triangle } n)^{2}$. The theorem is a consequence of (19).

(61) $\quad(\text { Triangle } n)^{2}+(\text { Triangle }(n+1))^{2}=$ Triangle $(n+1)^{2}$. The theorem is a consequence of (19).

(62) (Triangle $(n+1))^{2}-(\text { Triangle } n)^{2}=(n+1)^{3}$. The theorem is a consequence of (19).

(63) Let us consider a non zero natural number $n$. Then 3 . Triangle $n+$ Triangle $\left(n-^{\prime} 1\right)=\operatorname{Triangle}(2 \cdot n)$. The theorem is a consequence of (19).

(64) 3 . Triangle $n+\operatorname{Triangle}(n+1)=\operatorname{Triangle}(2 \cdot n+1)$. The theorem is a consequence of (19).

Let us consider a non zero natural number $n$. Now we state the propositions:

(65) (Triangle $\left(n-{ }^{\prime} 1\right)+6 \cdot$ Triangle $\left.n\right)+$ Triangle $(n+1)=8 \cdot$ Triangle $n+1$.

(66) Triangle $n+\operatorname{Triangle}\left(n-^{\prime} 1\right)=\frac{((1+2 \cdot n)-1) \cdot n}{2}$.

Now we state the propositions:

(67) $1+9 \cdot$ Triangle $n=$ Triangle $(3 \cdot n+1)$. The theorem is a consequence of (19).

(68) Let us consider a natural number $m$. Then Triangle $(n+m)=$ (Triangle $n+$ Triangle $m)+n \cdot m$. The theorem is a consequence of (19).

(69) Let us consider non trivial natural numbers $n, m$. Then Triangle $n$. Triangle $m+\operatorname{Triangle}\left(n-{ }^{\prime} 1\right) \cdot \operatorname{Triangle}\left(m-^{\prime} 1\right)=\operatorname{Triangle}(n \cdot m)$. The theorem is a consequence of (19). 


\section{Sets of Polygonal Numbers}

Let $s$ be a natural number. The functor PolyNum $s$ yielding a set is defined by the term

(Def. 9) the set of all Polygon $(s, n)$ where $n$ is a natural number.

Let $s$ be a non trivial natural number. Let us observe that the functor PolyNum $s$ yields a subset of $\mathbb{N}$. The functors: the set of all triangular numbers and the set of all square numbers yielding subsets of $\mathbb{N}$ are defined by terms, respectively.

(Def. 10) PolyNum 3.

(Def. 11) PolyNum 4.

Let $s$ be a non trivial natural number. Note that PolyNum $s$ is non empty and the set of all triangular numbers is non empty and the set of all square numbers is non empty and every element of the set of all triangular numbers is triangular and every element of the set of all square numbers is square.

Let us consider a number $x$. Now we state the propositions:

(70) $x \in$ the set of all triangular numbers if and only if $x$ is triangular.

(71) $x \in$ the set of all square numbers if and only if $x$ is square.

\section{Some Well-Known Properties}

Now we state the propositions:

(73) Triangle $n=\left(\begin{array}{c}n+1 \\ 2\end{array}\right)$. The theorem is a consequence of (72) and (19).

(74) Let us consider a non zero natural number $n$. If $n$ is even and perfect, then $n$ is triangular. The theorem is a consequence of (19). Proof: Consider $p$ being a natural number such that $2^{p}-^{\prime} 1$ is prime and $n=2^{p-^{\prime} 1} \cdot\left(2^{p}-^{\prime} 1\right)$. $p \neq 0$ by [21, (4)].

Let $n$ be a non zero natural number. Let us note that $M_{n}$ is non zero.

Let $n$ be a number. We say that $n$ is Mersenne if and only if

(Def. 12) There exists a natural number $p$ such that $n=M_{p}$.

Note that there exists a prime number which is Mersenne and there exists a natural number which is non prime and there exists a natural number which is Mersenne and non prime and every prime number is non zero.

Let $n$ be a Mersenne prime number. One can check that Triangle $n$ is perfect and every non zero natural number which is even and perfect is also triangular.

Now we state the propositions:

(75) $8 \cdot$ Triangle $n+1=(2 \cdot n+1)^{2}$. The theorem is a consequence of (19). 
(76) If $n$ is triangular, then $8 \cdot n+1$ is square. The theorem is a consequence of $(75)$.

(77) If $n$ is triangular, then $9 \cdot n+1$ is triangular. The theorem is a consequence of (67).

(78) If Triangle $n$ is triangular and square, then Triangle $((4 \cdot n) \cdot(n+1))$ is triangular and square. The theorem is a consequence of (19).

Let us observe that the set of all triangular numbers is infinite and the set of all square numbers is infinite and there exists a natural number which is triangular, square, and non zero.

Now we state the proposition:

(79) 0 is triangular and square.

Let us observe that every number which is zero is also triangular and square.

Now we state the proposition:

(80) 1 is triangular and square. The theorem is a consequence of (11).

Now we state the propositions:

(81) Square triangular number:

36 is triangular and square. The theorem is a consequence of (11), (80), (78), and (18).

(82) 1225 is triangular and square. The theorem is a consequence of (19).

Let $n$ be a triangular natural number. One can check that $9 \cdot n+1$ is triangular.

Let us note that $8 \cdot n+1$ is square.

\section{Reciprocals of TRIANgUlar Numbers}

Let $a$ be a real number. One can verify that $\lim \{a\}_{n \in \mathbb{N}}$ reduces to $a$.

The functor ReciTriang yielding a sequence of real numbers is defined by

(Def. 13) Let us consider a natural number $i$. Then $i t(i)=\frac{1}{\text { Triangle } i}$.

Let us note that (ReciTriang)(0) reduces to 0.

Now we state the propositions:

(83) $\frac{1}{\text { Triangle } n}=\frac{2}{n \cdot(n+1)}$. The theorem is a consequence of (19).

(84) $\left(\sum_{\alpha=0}^{\kappa}(\text { ReciTriang })(\alpha)\right)_{\kappa \in \mathbb{N}}(n)=2-\frac{2}{n+1}$. The theorem is a consequence of (83). Proof: Define $\mathcal{P}$ [natural number] $\equiv\left(\sum_{\alpha=0}^{\kappa}(\text { ReciTriang })(\alpha)\right)_{\kappa \in \mathbb{N}}\left(\$_{1}\right)$ $=2-\frac{2}{\$_{1}+1} \cdot \mathcal{P}[0]$. For every natural number $k$ such that $\mathcal{P}[k]$ holds $\mathcal{P}[k+1]$. For every natural number $k, \mathcal{P}[k]$ from $[2$, Sch. 2].

The functors: SumsReciTriang and $\operatorname{GeoSeq}(a, b)$ yielding sequences of real numbers are defined by conditions, respectively.

(Def. 14) Let us consider a natural number $n$. Then (SumsReciTriang) $(n)=2-$ $\frac{2}{n+1}$. 
(Def. 15) Let us consider a natural number $n$. Then $(\operatorname{GeoSeq}(a, b))(n)=\frac{a}{n+b}$.

Let $a, b$ be real numbers.

Now we state the propositions:

(85) Let us consider real numbers $a, b$. Suppose $b>0$. Then

(i) $\operatorname{GeoSeq}(a, b)$ is convergent, and

(ii) $\lim \operatorname{GeoSeq}(a, b)=0$.

(86) SumsReciTriang $=\{2\}_{n \in \mathbb{N}}+-\operatorname{GeoSeq}(2,1)$. Proof: For every natural number $k$, (SumsReciTriang) $(k)=\left(\{2\}_{n \in \mathbb{N}}\right)(k)+(-\operatorname{GeoSeq}(2,1))(k)$ by [19, (57)].

(87) (i) SumsReciTriang is convergent, and

(ii) $\lim$ SumsReciTriang $=2$.

The theorem is a consequence of (85) and (86).

(88) $\left(\sum_{\alpha=0}^{\kappa}(\text { ReciTriang })(\alpha)\right)_{\kappa \in \mathbb{N}}=$ SumsReciTriang.

Now we state the proposition:

(89) ReCiprocals of triangular numbers:

$\sum$ ReciTriang $=2$.

\section{REFERENCES}

[1] Kenichi Arai and Hiroyuki Okazaki. Properties of primes and multiplicative group of a field. Formalized Mathematics, 17(2):151-155, 2009. doi:10.2478/v10037-009-0017-7

[2] Grzegorz Bancerek. The fundamental properties of natural numbers Formalized Mathematics, 1(1):41-46, 1990.

[3] Grzegorz Bancerek. The ordinal numbers. Formalized Mathematics, 1(1):91-96, 1990.

[4] Grzegorz Bancerek and Krzysztof Hryniewiecki. Segments of natural numbers and finite sequences. Formalized Mathematics, 1(1):107-114, 1990.

[5] Czesław Byliński. Finite sequences and tuples of elements of a non-empty sets. Formalized Mathematics, 1(3):529-536, 1990.

[6] Czesław Byliński. Functions and their basic properties Formalized Mathematics, 1(1): 55-65, 1990.

[7] Czesław Byliński. Functions from a set to a set. Formalized Mathematics, 1(1):153-164, 1990.

[8] Czesław Byliński. Partial functions Formalized Mathematics, 1(2):357-367, 1990.

[9] Czesław Byliński. The sum and product of finite sequences of real numbers Formalized Mathematics, 1(4):661-668, 1990.

[10] Czesław Byliński. Some basic properties of sets Formalized Mathematics, 1(1):47-53, 1990.

[11] Agata Darmochwał. Finite sets. Formalized Mathematics, 1(1):165-167, 1990.

[12] Yuzhong Ding and Xiquan Liang. Solving roots of polynomial equation of degree 2 and 3 with complex coefficients Formalızed Mathematıcs, 12(2):85-92, 2004.

[13] Yoshinori Fuijsawa, Yasushi Fuwa, and Hidetaka Shimizu. Public-key cryptography and Pepin's test for the primality of Fermat numbers. Formalized Mathematıcs, $7(\mathbf{2}): 317-321$, 1998.

[14] Yuichi Futa, Hiroyuki Okazaki, Daichi Mizushima, and Yasunari Shidama. Operations of points on elliptic curve in proiective coordinates. Formalized Mathematics, 20(1):87-95, 2012. doi: $10.2478 / v 10037-012-0012-2$.

[15] Carl Friedrich Gauss. Disquisitiones Arithmeticae. Springer, New York, 1986. English translation. 
[16] Richard K. Guy. Every number is expressible as a sum of how many polygonal numbers? American Mathematical Monthly, 101:169-172, 1994.

[17] Thomas L. Heath. A History of Greek Mathematics: From Thales to Euclid, Vol. I. Courier Dover Publications, 1921.

[18] Andrzej Kondracki. Basic properties of rational numbers Formalized Mathematics, 1(5): 841-845, 1990.

[19] Jarosław Kotowicz. Real sequences and basic operations on them Formalized Mathematics, 1(2):269-272, 1990.

[20] Jarosław Kotowicz. Convergent sequences and the limit of sequences. Formalized Mathematics, 1(2):273-275, 1990.

[21] Rafał Kwiatek. Factorial and Newton coefficients Formalized Mathematics, 1(5):887-890, 1990.

[22] Rafał Kwiatek and Grzegorz Zwara. The divisibility of integers and integer relatively primes Formalized Mathematics, 1(5):829-832, 1990.

[23] Robert Milewski. Natural numbers Formalized Mathematics, 7(1):19-22, 1998.

[24] Adam Naumowicz. Conjugate sequences, bounded complex sequences and convergent complex sequences Formalızed Mathematıcs, 6(2):265-268, 1997.

[25] Konrad Raczkowski and Andrzej Nędzusiak. Series Formalized Mathematics, 2(4):449452, 1991.

[26] Marco Riccardi. The perfect number theorem and Wilson's theorem. Formalized Mathematics, 17(2):123-128, 2009. doi 10.2478/v10037-009-0013-y.

[27] Piotr Rudnicki and Andrzej Trybulec. Abian's fixed point theorem. Formalized Mathematics, 6(3):335-338, 1997.

[28] Andrzej Trybulec. On the sets inhabited by numbers Formalized Mathematics, 11(4): 341-347, 2003.

[29] Andrzej Trybulec and Czesław Byliński. Some properties of real numbers. Formalized Mathematics, 1(3):445-449, 1990.

[30] Michał J. Trybulec. Integers. Formalized Mathematics, 1(3):501-505, 1990.

[31] Zinaida Trybulec. Properties of subsets. Formalized Mathematics, 1(1):67-71, 1990.

[32] André Weil. Number Theory. An Approach through History from Hammurapi to Legendre. Birkhäuser, Boston, Mass., 1983.

[33] Freek Wiedijk. Formalizing 100 theorems

[34] Freek Wiedijk. Pythagorean triples $\mid$ Formalized Mathematics, 9(4):809-812, 2001.

[35] Edmund Woronowicz. Relations and their basic properties Formalized Mathematics, 1 (1):73-83, 1990.

Received May 19, 2013 